Viewpoint

\section{ROS-Induced DNA Damage as an Underlying Cause of Aging}

\author{
Gabriel Robert, J. Richard Wagner* \\ Département de médecine nucléaire et radiobiologie Faculté de médecine et des \\ sciences de la santé, Université de Sherbrooke, J1H 5N4, Sherbrooke, Canada \\ * Correspondence: J. Richard Wagner, \\ Email: Richard.J.Wagner@USherbrooke.ca.
}

\begin{abstract}
Oxidative or redox stress arises from the generation of reactive oxygen species (ROS) as a by-product of oxygen utilization. ROS-mediated damage to DNA accumulates over time and leads to irreversible changes in cellular functions and integrity associated with aging phenotypes. Here, we discuss the contribution of ROS-induced DNA damage to aging and the important role of deficiencies in DNA repair pathways that counteract this damage. We elaborate on the relationship of aging with mutagenesis and epigenetics and conclude with promising avenues to prevent aging.
\end{abstract}

KEYWORDS: reactive oxygen species; redox stress; DNA damage; DNA repair; mutagenesis; epigenetics; antiaging therapies

\section{GENERAL HYPOTHESIS}

Aging can be defined as a gradual and stochastic accumulation of errors over time that leads to cellular and tissue malfunction, physiological decline and accrued risks of age-related diseases. There is no single cause, mechanism or solution. This viewpoint article argues for DNA damage induced by reactive oxygen species (ROS) as a crucial contributor to aging. A common feature of theories of aging is oxidative stress (more appropriately called redox stress), which is omnipresent in organisms that depend on oxygen for life-support. We hypothesize that ROS-induced DNA damage increases with age due mainly to less efficient DNA repair. In some cases, continuous damage induces mutations particularly in rapidly dividing cells leading to an increase in the risk of age-related cancer, whereas in other cases, the inability to process and repair DNA damage leads to problems in replication and transcription leading to senescence and cell death. Alterations of epigenetic marks also contribute to changes in chromatin architecture and gene expression profiles. Accumulation of DNA damage and mutations can progressively engender deficiencies in the production of RNA and proteins, or the appearance of rogue-like RNA and aberrant protein molecules. Our understanding of aging is complicated by the synergistic action of multiple causes and the interconnectivity between the hallmarks of aging [1,2]. The stochastic 
nature of events leads to a diversity of cellular profiles that develop into aging phenotypes. Our ability to identify specific genetic traits, lifestyle practises and modulators of lifespan is compromised by the enormous variability of aging biomarkers and the inherent lack of a true control individual or experiment for comparison.

\section{ROS AND AGING}

ROS are constantly generated during aerobic metabolism mainly as a by-product of ATP-producing mitochondrial electron transport and targeted NADPH-dependent phagocytosis that contribute to inflammation. Examples of ROS include the superoxide radical anion $\left(\mathrm{O}_{2} \cdot^{-}\right)$and hydrogen peroxide $\left(\mathrm{H}_{2} \mathrm{O}_{2}\right)$, which reacts with redox active metal ions, such as $\mathrm{Fe}^{2+}$ and $\mathrm{Cu}^{+}$, to produce the highly reactive and deleterious hydroxyl radical $(\cdot \mathrm{OH})$ via Fenton-type reactions. Hypochlorous acid (HOCl), a chlorinating and oxidizing agent, is enzymatically generated by myeloperoxidase released from activated neutrophils during inflammation. Subsequent reactions of ROS lead to peroxyl radicals, hydroperoxides, alkoxyl radicals and singlet oxygen, which can greatly amplify damage to biomolecules. Taking kinetic and abundance data, highly damaging $\cdot \mathrm{OH}$ mainly react with proteins and nucleic acids (80\%) while small molecule antioxidants play a minor role $(<20 \%)$ [3]. Although ROS can efficiently inflict damage, they also serve as important signalling agents. From a recent count, over 40 enzymes in different compartments of the cells are known to generate ROS, controlling redox states, metabolic regulatory factors and stress responses [4]. Interestingly, $10-20 \%$ of cellular proteins are redoxsensitive as inferred by changes in the cysteine proteome and estimates may be higher upon further advances in proteomics [5]. Thus, a balance of ROS generators versus ROS defenders is critical, and disturbances, i.e., redox stress, can have profound effects on homeostasis, growth, and adaptive responses. There is overwhelming evidence that redox stress increases with advancing age based on an increase in oxidatively modified biomolecules, chronic activation of ROS-signalling pathways, and the effects of compounds that can modulate redox stress in cultured cells and rodents [6-11]. An exacerbation of ROS production may also be explained by age-related dysfunction in mitochondria due in part to the accumulation of DNA damage in these organelles as a central idea in the mitochondrial theory of aging. Lastly, state of redox stress is also exemplified by the expanding number of senescent cells in tissues with aging, which further drives ROS formation via the expression of a proinflammatory senescenceassociated secretory phenotype (SASP) [12-15].

\section{DNA DAMAGE AND AGING}

The integrity of the genome is continuously challenged by endogenous chemical alterations, such as spontaneous hydrolysis, ROS-mediated damage, and the formation of adducts with reactive aldehydes or alkylating agents. It has been approximated that as many as 100,000 
modifications of DNA are produced daily in various cellular genomes. ROS likely contribute in large part to this damage leading to the formation of a myriad of structurally different lesions. In particular, Fenton-like reactions generate $\cdot \mathrm{OH}$, which reacts with DNA to give about 20 major DNA base and sugar oxidation products, strand breaks, and DNA-protein crosslinks [16]. ROS-induced DNA damage, abasic sites and strand breaks, and downstream damage, such as chromosomal aberrations, have been shown to increase with aging $[10,11]$. Many studies have focused on 8-oxo7,8-dihydroguanine (80xoG) as a biomarker of ROS-induced DNA damage even though it represents a small percentage of the multitude of other lesions that have been characterized in model studies. Most of this work however is troubled by the problem of artefactual oxidation of DNA during sample preparation for analysis leading to an overestimation of damage [17]. In studies that minimize artefactual oxidation, the level of 8oxoG appears to be about 1 damage per million nucleotides and increases about 2-fold in most organs between young and older animals [10]. Nevertheless, it is imperative to identify and measure a more complete set of ROS-induced lesions in vivo because their structure ultimately determines the biological outcomes. For example, the fact that aging is a major risk factor for cancer suggests that accumulation of DNA damage is a central mechanism shared by both [18]. A comparison of the profile of various lesions in aging cells with those in cancer would greatly help one to pinpoint differences in the causes and consequences of DNA damage in these two conditions. There are several other assays of DNA damage, such as $\gamma \mathrm{H} 2 \mathrm{AX}$ for double strand breaks, qPCR- and comet-based assays; however, they give little information about the primary structure of DNA lesions that in turn is converted into breaks during DNA repair. Future studies should focus on developing robust assays, which minimize artefactual oxidation, and permit a greater coverage within the spectrum of DNA modifications.

\section{DNA REPAIR AND AGING}

The importance of DNA repair in aging is underscored by the existence of premature aging syndromes that present deficiencies in DNA repair pathways. [19-21]. These diseases are interesting because they accurately display many features of the aging phenotype and age-related pathologies in humans. More than 200 proteins, seven principle pathways and various damage sensors and response elements are implicated in DNA repair. The pathways of DNA repair depend on the structure and extent of DNA damage. Most ROS-induced damage is efficiently repaired by base excision repair (BER). Single strand breaks also generated during the initial step of BER are repaired by apyrimidinic/apurinic endonuclease (APE), a highly abundant DNA repair protein. Other repair pathways include nucleotide excision repair (NER) mostly for bulky lesions, mismatch repair (MMR) mostly for replication errors, and non-homologous end joining (NHEJ) and homologous recombination (HR) for double strand break repair. In 
general, the efficiency of repair decreases with age; for example, the activity of certain $N$-glycosylases, polymerase $\beta$ and ligase III of BER decreases with age [19,22]. In addition, aging phenotypes persist in knockout mice that lack critical components of DNA damage response (DDR) $[11,19]$. Depending on the structure and frequency of DNA damage, DNA repair processing can induce either mutagenesis that promotes cell survival and cancer or impede replication and transcription that promote cell death and aging. Interestingly, defects in transcriptional coupled (TC) repair observed in Ercc1 mutant mice display features of aging, which can be related to the accumulation of DNA damage resulting in stalled transcription complexes, particularly in long genes and in post-mitotic cells [23,24]. Another interesting example involves the induction of multiple telomere defects including telomere shortening, and in the long run, genetic instability, when $G$ is specifically oxidized to 80xoG in telomeres together with ablation of 8oxoG-specific DNA $N$-glycosylase OGG1 [25]. With future research, we may be able to identify the downstream effects of each protein and determine where the errors and deficiencies are most likely to occur during aging.

\section{MUTAGENESIS AND AGING}

The conversion of DNA damage into mutations occurs precipitously during cell division. If damage is not recognized as a cognate base by replicative polymerase, and depending on the extent of damage, alternative polymerases undergo error-prone translesion bypass synthesis to avoid deleterious stalling and collapse of the replication fork. In contrast, DNA damage can be converted into mutations in post-mitotic cells during DNA repair that occasionally induces errors and that probably worsens with aging. Thus, the accumulation of mutations is different in actively dividing cells and post-mitotic cells. It is clear today that all types of mutations accumulate with age in diverse cells, tissues and species, including simple point mutations, transpositions, rearrangements and chromosomal aberrations [19]. However, it is difficult to obtain a consistent mutation signature of aging because mutations are stochastic and heterogeneous and not likely to reach a high enough percentage in the population for analysis. In contrast, it is possible to identify driver genes that cause cancer because mutated genomes undergo clonal selection and become relatively homogeneous. In a recent article, numerous mutational signatures were extracted from various cancer genome databases and three signatures of unknown aetiology were present at high levels in most tumors and strongly associated with age [26]. Interestingly, these signatures contained a high percentage of $\mathrm{C}$ to $\mathrm{T}$ transitions. Although it is generally believed that these mutations arise from thermal or enzymatic induced deamination of cytosine to uracil, another important source may be through initial ROS-induced oxidation of cytosine, which renders thismolecule 6 to 7 orders of magnitude more susceptible to deamination [27]. The deamination of either cytosine (C) or 5-methylcytosine $(5 \mathrm{mC})$ 
appears to be an important common denominator of aging and cancer. For example, $\mathrm{C}$ to $\mathrm{T}$ transitions are the most common mutations in the genome representing $30 \%$ of single nucleotide polymorphisms (SNPs). The presence of SNPs in genome maintenance genes are associated with aging of the cardiovascular system and the incidence of cancer [28-30]. Surprisingly, mutations are 10-100 fold more frequent at methylated CpGs than at non-methylated CpGs [31-35], indicating that the deamination of $5 \mathrm{mC}$ and subsequent repair is a weakness in maintaining genome stability during aging.

\section{EPIGENETICS AND AGING}

Epigenetic alteration is a hallmark of aging. Epigenetics play a pivotal role in gene expression during development and later stages of life. These changes do not involve modifications in the genetic code but rather the reversible addition of small chemical groups, such as methyl groups, onto the structure of DNA and associated histones; in addition, non-coding RNA and modifications of RNA contribute to gene expression. The methylation of $\mathrm{C}$ to $5 \mathrm{mC}$ occurs extensively at $\mathrm{CpG}$ dinucleotides resulting in selective and reversible gene expression. This process representing 5\% of nonmodified cytosines has the capacity to alter the expression of thousands of genes. An individual's methylation pattern is unique while certain commonalities have been considered good predictors of chronological age. In general, there is a global loss of $5 \mathrm{mC}$ (i.e., hypomethylation) across the genome during aging, accompanied with site-specific hypermethylation. High CpG density and the ability to relocate chromatin modifiers confer chromatin stability and correlates with species lifespan [36]. Recently, a novel pathway was discovered that reverses the methylation of cytosine, involving the enzymatic oxidation of $5 \mathrm{mC}$ to 5-hydroxymethylcytosine (5hmC) by TET family [37]. The latter modification is especially abundant in pluripotent cells as well as in post-mitotic brain cells, where it reaches as much as $1 \%$ of non-modified cytosine. Pathophysiological changes in the genetic pattern of $5 \mathrm{hmC}$ are more dramatic than $5 \mathrm{mC}$ [38]. $5 \mathrm{hmC}$ is also a lesion arising from the oxidation of $5 \mathrm{mC}$ [37], and thus, it is possible that ROS drive epigenetic changes through the accumulation of damage and mutations at methylated CpGs leading to hypomethylation and expression of DNA that is detrimental to survival.

\section{PREVENTION OF AGING}

How can one delay aging and extend lifespan? Since the steady-state level of DNA damage is an equilibrium, either reducing ROS-induced damage or improving DNA repair are feasible strategies. Apart from a few exceptions $[39,40]$, the possibility of reducing damage by augmenting antioxidants defenses has met with inconsistent results toward preventing aging, either via the modulation of antioxidant enzyme expression or administration of an excess of natural or exogenous compounds [41,42]. This is probably due in part to the importance of ROS in signalling 
pathways [4] and the likelihood that cells adapt to antioxidant-induced changes in ROS. A common problem with antioxidants is that they cannot reach a high enough concentration in cells to effectively scavenge all reactive radicals, especially $\mathrm{OH}$. Part of the effectiveness of caloric restriction (CR), which is viewed as the most efficient antiaging therapy so far, has been attributed to a reduction in oxidative damage, but the implication of endogenous antioxidants is unclear [8,43,44]. The effect of CR in mice carrying mutant DNA repair genes can triple lifespan, indicating a strong link between aging and DNA repair [23]. Indeed, CR can be explained in part by a metabolic switch from growth to genome maintenance the socalled survival response [45]. There is much recent interest in the systemic destruction of senescent cells to reduce the burden of chronic oxidative stress that damages surrounding cells via SASP [46-48]. This approach shows much promise in alleviating certain aging-associated phenotypes. Two compounds, quercetin and dasatinib, were shown to afford substantial senolytic effects, physiological improvements and lifespan extension. Nevertheless, we believe a better overall strategy would be to tip the balance toward genome maintenance. This approach should help preserve both post-mitotic cells (e.g., brain tissue) and stem cells by improving DNA repair preferably via the modulation of upstream regulators [22]. A significant part of CR protective effects are probably mediated by such mechanisms [43,49]. For example, CR led to the identification of the $\mathrm{NAD}^{+}$-dependent deacylase and ADPribosyltransferase enzymes called sirtuins as possible lifespan regulators. Related and recent studies have demonstrated that sirtuins play a key role in DNA repair, maintenance of genome integrity, as well as in the regulation of responses to redox stress [50-54]. $\mathrm{NAD}^{+}$levels decline with age and supplementation of $\mathrm{NAD}^{+}$intermediates is currently under review to replenish and activate sirtuins. This might also stabilize the function of poly-(ADP-ribose) polymerases (PARP) enzymes, which are involved in DNA repair and compete for $\mathrm{NAD}^{+}$stocks [55-57]. Alternatively, sirtuinactivating compounds (STACs) could be designed to promote the upstream pathways of DNA repair [58]. Another approach is to increase genome maintenance by artificially promoting the DDR signaling cascade, as proposed by Gioia et al. [59], although caution should be taken because, paradoxically, chronic DDR activation can accelerate aging by inducing senescence. In the future, we will need to engage in more intense and multidisciplinary research to fully understand the biochemical and biological steps of aging and move forward to develop plausible strategies that prevent the underlying causes.

\section{CONFLICTS OF INTEREST}

The authors declare that they have no conflicts of interest 


\section{FUNDING}

Our work is supported by a grant from the Natural Science and Engineering Research Council of Canada (NSERC: RGPIN-2016-05124). We thank the "Centre de Recherche sur le Vieillissement" and "Fonds de la Recherche en Santé du Québec” for infrastructure support.

\section{REFERENCES}

1. López-Otín C, Blasco MA, Partridge L, Serrano M, Kroemer G. The hallmarks of aging. Cell. 2013;153(6):1194.

2. Liochev SI. Which is the most significant cause of aging? Antioxidants. 2015;4(4):793-810.

3. Davies MJ. The oxidative environment and protein damage. Biochim Biophys Acta Proteins Proteomics. 2005;1703(2):93-109.

4. Sies H, Jones DP. Reactive oxygen species (ROS) as pleiotropic physiological signalling agents. Nat Rev Mol Cell Biol. 2020;21(7):363-83. doi: 10.1038/s41580-020-0230-3

5. Go Y, Chandler JD, Jones DP. The cysteine proteome. Free Radic Biol Med. 2015;84:227-45.

6. Barja G. Rate of generation of oxidative stress-related damage and animal longevity. Free Radic Biol Med. 2002;33(9):1167-72.

7. Ugarte N, Petropoulos I, Friguet B. Oxidized mitochondrial protein degradation and repair in aging and oxidative stress. Antioxidants Redox Signal. 2010;13(4):539-49.

8. Kregel KC, Zhang HJ. An integrated view of oxidative stress in aging: Basic mechanisms, functional effects, and pathological considerations. Am J Physiol Regul Integr Comp Physiol. 2007;292(1):18-36.

9. Stadtman ER. Protein oxidation and aging. Free Radic Res. 2006;40(12):1250-8.

10. Møller P, Løhr M, Folkmann JK, Mikkelsen L, Loft S. Aging and oxidatively damaged nuclear DNA in animal organs. Free Radic Biol Med. 2010;48(10):1275-85.

11. McNeely T, Leone M, Yanai H, Beerman I. DNA damage in aging, the stem cell perspective. Hum Genet. 2020;139(3):309-31.

12. Campisi J. Aging, cellular senescence, and cancer. Annu Rev Physiol. 2013;75:685-705.

13. Van Deursen JM. The role of senescent cells in ageing. Nature. 2014;509(7501):439-46.

14. Lasry A, Ben-Neriah Y. Senescence-associated inflammatory responses: Aging and cancer perspectives. Trends Immunol. 2015;36(4):217-28.

15. Freund A, Orjalo AV, Desprez PY, Campisi J. Inflammatory networks during cellular senescence: causes and consequences. Trends Mol Med. 2010;16(5):238-46.

16. Cadet J, Wagner JR. DNA base damage by reactive oxygen species, oxidizing agents, and UV radiation. Cold Spring Harb Perspect Biol. 2013;5:1-18.

17. Cadet J, Di Mascio P, Wagner JR. Radiation-induced (5'R)-and (5'S)-purine $5^{\prime}, 8$ cyclo-2'-deoxyribonucleosides in human cells: a revisited analysis of HPLC- 
MS/MS measurements. Free Radic Res. 2019;53(5):574-7.

18. De Magalhães JP. How ageing processes influence cancer. Nat Rev Cancer. 2013;13(5):357-65.

19. Moskalev AA, Shaposhnikov MV, Plyusnina EN, Zhavoronkov A, Budovsky A, Yanai $\mathrm{H}$, et al. The role of DNA damage and repair in aging through the prism of Koch-like criteria. Ageing Res Rev. 2013;12(2):661-84.

20. Best BP. Nuclear DNA Damage as a Direct Cause of Aging. Rejuvenation Res. 2009;12(3):199-208.

21. Carrero D, Soria-Valles C, López-Otín C. Hallmarks of progeroid syndromes: Lessons from mice and reprogrammed cells. DMM Dis Model Mech. 2016;9(7):719-35.

22. Gorbunova V, Seluanov A, Mao Z, Hine C. Changes in DNA repair during aging. Nucleic Acids Res. 2007;35(22):7466-74.

23. Vermeij WP, Dollé MET, Reiling E, Jaarsma D, Payan-Gomez C, Bombardieri $\mathrm{CR}$, et al. Restricted diet delays accelerated ageing and genomic stress in DNArepair-deficient mice. Nature. 2016;537(7620):427-31.

24. Milanese C, Bombardieri CR, Sepe S, Barnhoorn S, Payán-Goméz C, Caruso D, et al. DNA damage and transcription stress cause ATP-mediated redesign of metabolism and potentiation of anti-oxidant buffering. Nat Commun. 2019;10(1):4887.

25. Fouquerel E, Barnes RP, Uttam S, Watkins SC, Bruchez MP, Opresko PL. Targeted and Persistent 8-Oxoguanine Base Damage at Telomeres Promotes Telomere Loss and Crisis. Mol Cell. 2019;75(1):117-30.e6.

26. Alexandrov LB, Kim J, Haradhvala NJ, Huang MN, Tian Ng AW, Wu Y, et al. The repertoire of mutational signatures in human cancer. Nature. 2020;578(7793):94-101.

27. Grand A, Cadet J, Eriksson LA, Labet V, Jorge NL, Schreiber ML, et al. Comparison of the mechanism of deamination of 5,6-dihydro-5methylcytosine with other cytosine derivatives. Theor Chem Acc. 2012;131(4):1-11.

28. Durik M, Kavousi M, Van Der Pluijm I, Isaacs A, Cheng C, Verdonk K, et al. Nucleotide excision DNA repair is associated with age-related vascular dysfunction. Circulation. 2012;126(4):468-78.

29. Verschuren JJW, Trompet S, Deelen J, Stott DJ, Sattar N, Buckley BM, et al. NonHomologous End-Joining Pathway Associated with Occurrence of Myocardial Infarction: Gene Set Analysis of Genome-Wide Association Study Data. PLoS One. 2013;8(2):e56262.

30. Bautista-Niño PK, Portilla-Fernandez E, Vaughan DE, Danser AHJ, Roks AJM. DNA damage: A main determinant of vascular aging. Int J Mol Sci. 2016;17(5):1-26.

31. Zhang S, Glickman BW, De Boer JG. Spontaneous mutation of the lacI transgene in rodents: Absence of species, strain, and insertion-site influence. Environ Mol Mutagen. 2001;37(2):141-6.

32. Schaaper RM, Dunn RL. Spontaneous mutation in the Escherichia coli lacI gene. Genetics. 1991;129(2):317-26.

33. Vermulst M, Bielas JH, Kujoth GC, Ladiges WC, Rabinovitch PS, Prolla TA, et 
al. Mitochondrial point mutations do not limit the natural lifespan of mice. Nat Genet. 2007;39(4):540-3.

34. Cooper DN, Youssoufian H. The CpG dinucleotide and human genetic disease. Hum Genet. 1988;78(2):151-5.

35. Greenblatt MS, Bennett WP, Hollstein M, Harris CC. Mutations in the p53 Tumor Suppressor Gene: Clues to Cancer Etiology and Molecular Pathogenesist. Cancer Res. 1994;54:4855-78.

36. Bertucci EM, Parrott BB. Is CpG Density the Link between Epigenetic Aging and Lifespan? Trends Genet. 2020;36(10):725-727. doi: 10.1016/j.tig.2020.06.003

37. Cadet J, Wagner JR. TET enzymatic oxidation of 5-methylcytosine, 5hydroxymethylcytosine and 5-formylcytosine. Mutat Res Genet Toxicol Environ Mutagen. 2014;764-5:18-35.

38. López V, Fernández AF, Fraga MF. The role of 5-hydroxymethylcytosine in development, aging and age-related diseases. Ageing Res Rev. 2017;37:28-38.

39. Liebert MA, Mitsui A, Hamuro J, Nakamura H, Kondo N, Hirabayashi Y, et al. Overexpression of Human Thioredoxin in Transgenic Mice Controls Oxidative Stress and Life Span. Antioxid Redox Signal. 2002;4(4):693-6. doi: 10.1089/15230860260220201

40. Schriner SE, Linford NJ, Martin GM, Treuting P, Ogburn CE, Emond M, et al. Extension of murine life span by overexpression of catalase targeted to mitochondria. Science. 2005;308(5730):1909-11.

41. Muller FL, Lustgarten MS, Jang Y, Richardson A, Van Remmen H. Trends in oxidative aging theories. Free Radic Biol Med. 2007;43(4):477-503.

42. Sadowska-Bartosz I, Bartosz G. Effect of antioxidants supplementation on aging and longevity. Biomed Res Int. 2014;2014:404680. doi: 10.1155/2014/404680

43. Walsh ME, Shi Y, Van Remmen H. The effects of dietary restriction on oxidative stress in rodents. Free Radic Biol Med. 2014;66:88-99.

44. Hursting SD, Lavigne JA, Berrigan D, Perkins SN, Barrett JC. Calorie Restriction, Aging, and Cancer Prevention: Mechanisms of Action and Applicability to Humans. Annu Rev Med. 2003;54:131-52.

45. Garinis GA, van der Horst GTJ, Vijg J, Hoeijmakers JHJ. DNA damage and ageing: New-age ideas for an age-old problem. Nat Cell Biol. 2008;10(11):1241-7.

46. Xu M, Pirtskhalava T, Farr JN, Weigand BM, Palmer AK, Weivoda MM, et al. Senolytics improve physical function and increase lifespan in old age. Nat Med. 2018;24(8):1246-56.

47. Roos CM, Zhang B, Palmer AK, Ogrodnik MB, Pirtskhalava T, Thalji NM, et al. Chronic senolytic treatment alleviates established vasomotor dysfunction in aged or atherosclerotic mice. Aging Cell. 2016;15(5):973-7.

48. Chang J, Wang Y, Shao L, Laberge RM, Demaria M, Campisi J, et al. Clearance of senescent cells by ABT263 rejuvenates aged hematopoietic stem cells in mice. Nat Med. 2016;22(1):78-83.

49. Cabelof DC, Yanamadala S, Raffoul JJ, Guo ZM, Soofi A, Heydari AR. Caloric restriction promotes genomic stability by induction of base excision repair and reversal of its age-related decline. DNA Repair. 2003;2(3):295-307. 
50. Morris BJ. Seven sirtuins for seven deadly diseases of aging. Free Radic Biol Med. 2013;56:133-71.

51. Lagunas-Rangel FA. Current role of mammalian sirtuins in DNA repair. DNA Repair. 2019;80(2508):85-92.

52. Beauharnois JM, Bolívar BE, Welch JT. Sirtuin 6: A review of biological effects and potential therapeutic properties. Mol Biosyst. 2013;9(7):1789-806.

53. Webster BR, Lu Z, Sack MN, Scott I. The role of sirtuins in modulating redox stressors. Free Radic Biol Med. 2012;52(2):281-90.

54. Merksamer PI, Liu Y, He W, Hirschey MD, Chen D, Verdin E. The sirtuins, oxidative stress and aging: An emerging link. Aging. 2013;5(3):144-50.

55. Yoshino J, Baur JA, Imai S-I. NAD ${ }^{+}$Intermediates: The Biology and Therapeutic Potential of NMN and NR. Cell Metab. 2018;27(3):513-28.

56. Imai S-I, Guarente L. NAD ${ }^{+}$and sirtuins in aging and disease. Trends Cell Biol. 2014;24(8):464-71.

57. Cantó C, Menzies KJ, Auwerx J. NAD ${ }^{+}$Metabolism and the Control of Energy Homeostasis: A Balancing Act between Mitochondria and the Nucleus. Cell Metab. 2015;22(1):31-53.

58. Bonkowski MS, Sinclair DA. Slowing ageing by design: The rise of $\mathrm{NAD}^{+}$and sirtuin-activating compounds. Nat Rev Mol Cell Biol. 2016;17(11):679-90.

59. Gioia U, Francia S, Cabrini M, Brambillasca S, Michelini F, Jones-Weinert CW, et al. Pharmacological boost of DNA damage response and repair by enhanced biogenesis of DNA damage response RNAs. Sci Rep. 2019;9(1):1-15.

How to cite this article:

Robert G, Wagner JR. ROS-Induced DNA Damage as an Underlying Cause of Aging. Adv Geriatr Med Res. 2020;2(4):e200024. https://doi.org/10.20900/agmr20200024 